\title{
Microbial Symbionts and Sponge Heterotrophy; Morphological Aspects of Sponge: Symbiont Integration via SEM Analysis
}

\author{
E. Poppell, C. Marks, A. Hill, M. Hill \\ Department of Biology, University of Richmond, Richmond, VA 23173
}

The ecological and evolutionary relationship between sponges and their symbiotic microflora remains poorly understood, which limits our ability to understand broad scale patterns in benthicpelagic coupling on coral reefs. Sponges can process $>40 \mathrm{ml}$ of water per $\mathrm{kg}$ of tissue per hour, removing microbes and dissolved organic matter during the process. Some sponge species are known to harbor extraordinarily dense populations of microbes (high microbial abundance (HMA) sponges), while other species maintain bacterial populations at very low levels (low microbial abundance sponges (LMA)). HMA and LMA sponges have significantly different pumping rates and trophic states. The role these different feeding strategies play in benthic-pelagic coupling on coral reefs may be significant. Previous work in this area examined the importance of sponge pumping rates. It has been suggested that the dense tissue in the HMA sponges would result in smaller water canals, which would contribute to a decrease in water flow [1]. Previous work has confirmed these predictions given that HMA sponges displayed a slower pumping rate than LMA sponges [2]. The differences in microbial abundance suggested that there is a morphological explanation for the differences in pumping rate, which also provides evidence of significant differences between HMA and LMA sponge morphology and physiology.

To fully understand the role HMA and LMA sponges play on coral reefs, we have examined, in great detail, the structure and stability of the microbial community harbored by sponges, and the nature of the pumping apparatus, the choanocyte chamber (Fig. 1). This research proposes to examine aspects of choanocyte chamber morphology as it relates to the symbiotic status of the sponge. Our focus was to compare choanocyte chamber densities of HMA and LMA sponges to test the hypothesis that microbial abundance influences the evolution of choanocyte chamber characteristics.

To examine choanocyte chamber morphology, tissue samples are prepared for freeze-fracture and examined using Scanning Electron Microscopy (SEM) methodology by [3]. Images from the SEM work are used to quantify choanocyte chamber size and density. The analysis of choanocyte chamber densities was contrasted with pumping rates determined for HMA and LMA sponge species [2].

The proposed research examining aspects of the dynamics of sponge-microbe associations has important ramifications for our understanding of marine community structure and function. Through this research, we will gain insights into morphological and physiological aspects of different sponge species and their microfloral symbionts. We are interested in determining whether bacterial symbionts influence morphological, physiological, or ecological differences among a variety of sponge species. We will also begin to understand how these relationships might change under changing environmental scenarios. Effects of environmental change may alter microbial communities and ultimately disrupt the ability for sponges to function normally. 
Thus, this research will be of interest to scientists who manage coral reef ecosystems as we consider likely consequences of climate change. This project will enhance our understanding of some of these issues.

\section{References}

[1] S. Vogel Journal Exp. Biol. (1978) 76.

[2] Weisz et al Oecologia (2008) 155:367-376.

[3] Johnston \& Hildeman Mar.Biol. (1982) 67: 1-7.
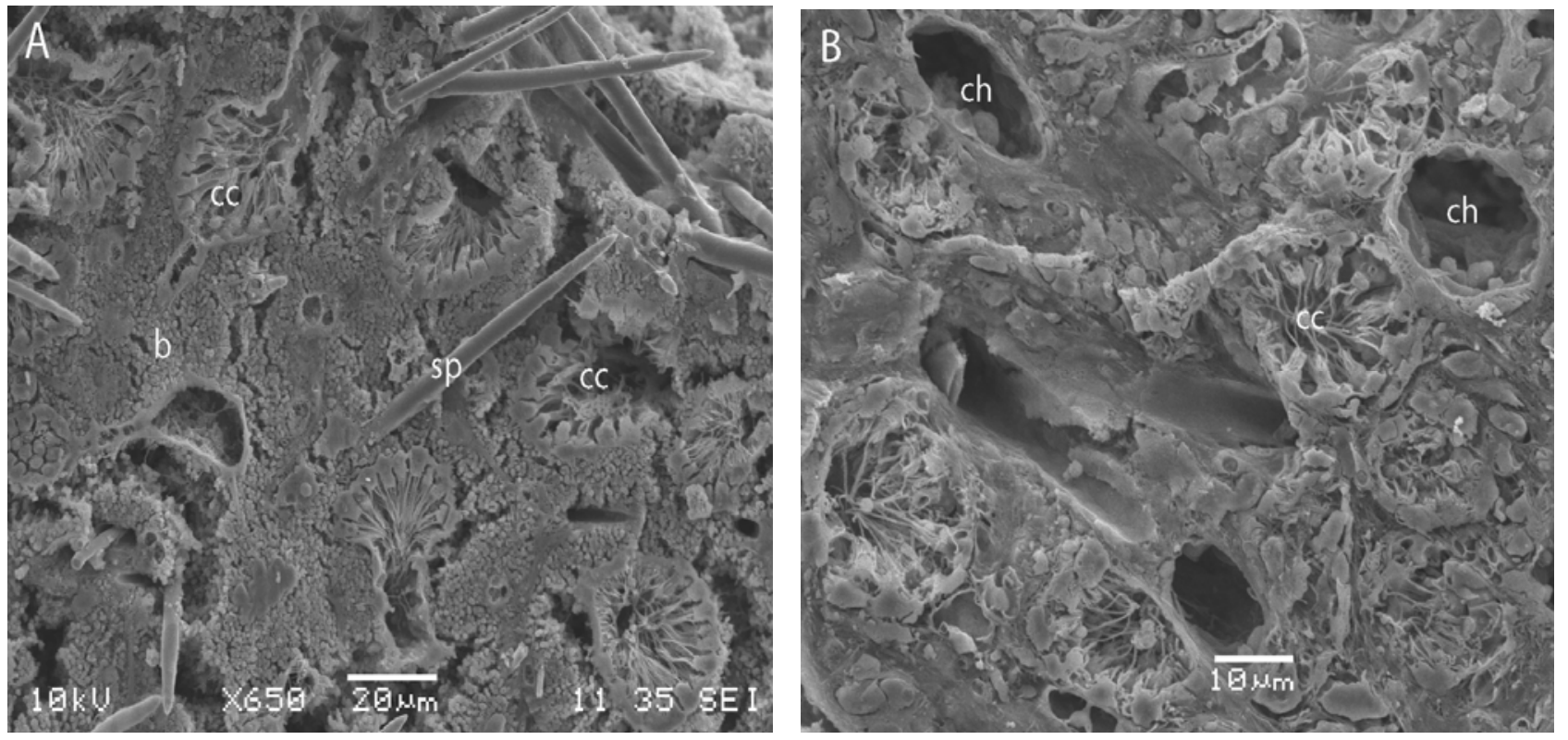

Figure 1. SEM micrograph of two tropical sponge species. Shown are the tissues of A Calyx podatypa $(\mathrm{HMA})$ and $\mathbf{B}$ Spheciospongia vesparium $(\mathrm{HMA}) . \mathrm{b}=$ bacteria, $\mathrm{ch}=$ channel, $\mathrm{cc}=$ choanocyte chambers (site of sponge feeding), $\mathrm{sp}=$ spicule. 